\title{
Damage Control Strategy
}

\section{Abdelkader Boukerrouche}

Department of Digestive Surgery, Hospital of Beni-Messous, University of Algiers, Algiers, Algeria

*Corresponding Author: Abdelkader Boukerrouche Department of Digestive Surgery, Hospital of Beni-Messous, University of Algiers, Algiers, Algeria.

Received: July 08, 2019; Published: July 26, 2019

DOI: $10.31080 /$ ASGIS.2019.02.0065

\section{Abstract}

Damage control surgery (DCS) includes principles of controlling bleeding; contain abdominal contamination, and completing definitive surgery on resuscitated patients. Evolved from DCS research, damage control resuscitation (DCR) incorporates early and massive blood product transfusion, restriction of crystalloid administration and permissive hypotension in selected patients. The development of DCS and DCR forms the current great achievement of modern trauma care. DCS combined with DCR has increasingly improved the survival in critically injured patients.

Keywords: Damage Control Surgery; Damage Control Resuscitation; Permissive Hypotension; Open Abdomen

\section{Introduction}

The bleeding is the major cause of death in trauma injury [1]. Performing repair surgery in severely injured patients with physiologic derangement is known to be detrimental to the outcome [2-4]. Therefore, rapid and effective control of bleeding associated with delayed definitive surgery and patient resuscitation was the best treatment strategy that led to improving outcome and survival in critically injured patients [2-4]. The continued research in trauma care has led to the development of new concepts in managing severely trauma patients [3]. These concepts include damage control surgery (DCS) and damage control resuscitation (DCR) and form the modern trauma care of critically injured patients [3]. The combined use of DCS and DCR has increasingly improved patient survival.

\section{Damage control surgery}

The damage control surgery concept (DCS) had been rapidly endorsed by the surgical emergency community as a treatment strategy for critically trauma injured patients [3]. The fundamental objective of the DCS strategy was to rapidly controlling bleeding, containing abdominal contamination, delaying repair surgery, restoring patient physiology, correcting coagulopathy and achieving a temporary abdominal wall closure. The definitive surgery will be completed with fascial closure if possible on optimized patients [4-7]. Historically, the concept of DCS strategy emerged from the clinical benefits of liver trauma management development $[8,9]$. In severely injured patients, the perihepatic packing has been firstly described in $1970[8,9]$. This treatment strategy aimed to control bleeding, delaying repair surgery, allowing patient resuscitation, correcting coagulopathy and performing definitive surgery on resuscitated patients. Over time, due to the important benefits of patient survival, the staged laparotomy with perihepatic packing has become a widespread practice and life-saving method in severe liver trauma [8-12].The benefits of the DCS strategy on survival has been clearly demonstrated in severely injured patients when applied in an appropriate setting $[5,13,14,15,16]$. However, this treatment approach is associated with increased health care resources utilization, high costs and prolonged length of ICU and hospital stays [16]. Also, the patients treated with DCS are subjected to multiple iterative surgeries. Therefore, it is so crucial to select the right patients within the appropriate scenarios .unfortunately, with lack of consensus, concerns remain regarding the appropriateness of the DCS indications. As a result, the rapid and widespread use of this approach has led to overutilization resulting in increased associated morbidity [17 ].In essence, the DCS approach should be considered in patients who continually suffer the tissue shock sequelae manifesting as persistent hypothermia and metabolic acidosis associated with non-surgical bleeding. Hence, opinion -based DCS triggers have been established including core temperature $<358 \mathrm{C}, \mathrm{pH}<7.2$, base deficit $>15$, and/ 
or significant coagulopathies [18]. However, with the lack of coherent research, these indications have not been validated [18]. Consequently, the most indications for DCS strategy are based on intraoperative rather than preoperative data including injured patient characteristics such as physiology, injuries, amount or type of provided resuscitation [18]. So as a result of large volume experience, not all patients initially presenting with physiology derangements require DCS process, patients with multiple abdominal injuries do not always engage in metabolic failure and with rapid control of bleeding and intensive resuscitation, some patients improve dramatically their physiologic parameters allowing repair surgery.

\section{Damage control resuscitation}

The patient resuscitation is considered as a crucial step of the damage control surgery strategy. The continued research on trauma care resulted in the development of a new concept "damage control resuscitation "(DCR). This concept incorporates early blood product administration with massive transfusion and early correction of coagulopathy, restriction of fluid administration, permissive hypotension in selected patients, and rapid and immediate control of bleeding. This resuscitation strategy must be started in the emergency room and continued intraoperatively and postoperatively in the IUC [19]. Compared to traditional resuscitation, DCR allows rapid and early correction of physiological derangements and post-traumatic bloody vicious cycle as well as it increases the rate of repair surgery during the first operation [19-22]. Also, correctly implemented, DCR reduced the need for DCS strategy, decreased the stay length in the ICU, increased the rate of primary abdominal closure [22-25]. Currently, the DCR combined with DCS form the modern trauma care continuum $[26,27]$.

\section{Permissive hypotension}

The dogma of restoring the physiologic blood pressure of patient has been challenged by describing the concept of permissive hypotension in 1994 [27]. This concept criticized initially, has been recently supported by the published report [28]. Therefore, Permissive hypotension is a major topic of recent trauma research [29]. The hypotensive resuscitation aims to decrease bleeding from injured tissue or the operative site. The Short-term hypotension with a mean arterial pressure of $50 \mathrm{mmHg}$ or systolic blood pressure of $80-100 \mathrm{mmHg}$ is well tolerated in non-severe bleeding; however, aggressive resuscitation should be undertaken in patients whose conditions continue to deteriorate $[28,29]$. The conservative hypotensive strategy is more efficacious resuscitation method to facilitate the complete control of bleeding with less administration of blood components and crystalloids, decrease postoperative coagulopathy and reduce early mortality [28,30-33]. Moreover, hypertensive resuscitation has been revealed to be a more effective strategy than traditional resuscitation in terms of multi-organ failure and acute respiratory distress syndrome [33]. Therefore, permissive hypotension can be applied in critically injured patients who respond to resuscitation or need transfer to the trauma centre, and in the preoperative setting Moreover, permissive hypotension can be used preoperatively and perioperatively; however, once bleeding controlled, the blood pressure should be maintained to a normal level with sufficient urine output. Additionally, if the time to control bleeding is short, the indication of permissive hypotension becomes obvious resulting in reduced blood transfusion. Unfortunately, there is no current consensual strategy regarding when and how to apply permissive hypotension during the resuscitation phase, and its duration and tolerability, Hence, further studies investigating these issues are highly needed.

\section{Abdominal Compartment Syndrome}

Abdominal compartment syndrome is characterized by an abdominal pressure superior to $20 \mathrm{mmHg}$ with organ dysfunction/ failure [34-38]. Oppositely, the intra-abdominal hypertension is defined as a pression elevation greater than $12 \mathrm{mmHg}$ and it is graded from I $-V$. The ACS syndrome has been first, described after abdominal closure in 1993[35]. A tightly packed and closed abdomen associated with intestinal oedema led to significantly increasing the intra-abdominal pressure and the development of ACS) [39]. The ACS impacts the major system including cardiovascular (hypotension), renal (acute kidney injury) and respiratory (failure). Fascinatingly, the risk factors for developing ACS as the same as those proposed as physiologic triggers for indicating DCS/DCR. Indeed, these indicators $(\mathrm{pH}$, base deficit, and core temperature) clearly represent the extremely physiologic derangements of most critically injured patients. Initially, the reported ACS incidence was superior to $30 \%$ and associated mortality was greater than $60 \%$ in major trauma patients [15]. However, over time, open abdomen surgery combined to DCR technique has increasingly reduced the incidence of ACS syndrome $[35,36,38]$. Therefore, temporary abdomen closure and planned delayed fascial closure is a vital component of DCS strategy and a clear method to prevent ACS. Currently, the prevention of ACS constitutes the greatest documented achievement of modern post injury critical care. 
Open Abdominal or temporary abdominal closure

Open abdomen (OA) or temporary abdominal closure (TAC) is a planned management strategy applied in critically injured patients who need relaparotomy to complete definitive surgery $[41,42]$. So, the abdominal organs are covered with skin or prosthetic materials to protect them and avoid abdominal pollution $[41,42]$. Historically, delaying primary abdominal wall closure in the context of DCS approach has been described firstly in 1981 [43]. Compared to delayed fascial closure, the mortality was higher in patients whose primary abdominal wall was closed under tension resulting in intra-abdominal pression elevation and abdominal compartment syndrome (ACS) [43]. Multiple techniques are available for covering the open abdomen including the skin closure techniques include skin suture, towel clips, silo technique and silicone diaphragm placement. The skin closure technique is rapid, economical and easy to implement. However, it is associated with increased risk of skin necrosis, abdominal contamination, evisceration and abdominal wall retraction. Hence, this technique is rarely used nowadays. Fascial closure techniques (FCTs) consist of suturing prosthetic materials (absorbable and nonabsorbable meshes) to the edges of the abdominal fascia in order to cover and protect the abdominal organs [44]. The materials sutured to fascia edges should be redundant to keep abdominal wall relax and prevent ACS, and progressively tightened in the postoperative stage $[44,45]$. The great achievement of FCTs is to obtain a tension-free temporary abdominal closure (TAC), facilitate repeated operations especially for patients who have limited opportunity to perform definitive abdominal closure within 1 week $[44,45]$. The disadvantages of the FCTs are the inability of wound drainage and peritoneal fluid evacuation that can lead to recurrent ACS. Another major inconvenient with the use of nonabsorbable mesh is the risk of intestinal fistula formation [44-46]. Vacuum-assisted closure technique consists of covering the exposed intestine with omentum majus and suturing tailored gelatin sponge composite material to the abdominal wall in order to provide a moist environment and avoid intestinal desiccation. A biological membrane is used to seal foam and wounds with 3-4 cm over the incision edge. The applied negative pressure varies from 45-60 mmHg. Besides, vacuumassisted closure system prevents mechanical damage of viscera, facilitates peritoneal fluid evacuation, avoids contamination of abdominal cavity, reduces inta-abdominal pression (IAP), decreases dressing change number, and favours wound healing $[44,46,47]$. Among previously described techniques for covering OA, The vacuum-assisted closure system is the most used technique with multiple choices $[44,46,47]$. However, a trauma centre should use a single technique that is more familiar with the health care team including surgeons and nurses working in the ICU. The OA approach has increasingly contributed to preventing ACS following DCS strategy. Unfortunately, the OA is associated with significant early morbidities such as protein and fluid loss, sepsis, entero-atmospheric fistula, overuse of care health resources and high economic costs $[48,49]$. As long-term morbidity, OA is a source of chronic physical discomfort, delayed return to work and poor quality of life $[48,49]$. Over time, as a result of continued research on trauma care, the OA approach has become a component of DCS strategies for trauma management.

\section{Conflict of Interest}

None declared.

\section{Bibliography}

1. Kauvar DS., et al. "Impact of hemorrhage on trauma outcome: an overview of epidemiology, clinical presentations, and therapeutic considerations". Journal of Trauma 60.6 (2006): S3S11.

2. Mikhali J. "The trauma triad of death: hypothermia, acidosis and coagulopathy". ACCN Clinical Issues 10.1 (1999): 85-94.

3. Chovanes J., et al. "The evolution of damage control surgery". Surgical Clinics of North America 92.4 (2012): 859-875.

4. Moore EE. "Thomas G. Orr Memorial Lecture. Staged laparotomy for the hypothermia, acidosis, and coagulopathy syndrome". The American Journal of Surgery 172.5 (1996): 405410 .

5. Rotondo MF., et al. "Damage control': an approach for improved survival in exsanguinating penetrating abdominal injury". Journal of Trauma 35.3 (1993): 375-382.

6. Rotondo MF and Zonies DH. "The damage control sequence and underlying logic". Surgical Clinics of North America 77.4 (1997): 761-777.

7. Stone HH., et al. "Management of the major coagulopathy with onset during laparotomy". Annals of Surgery 197.5 (1983): 532-535.

8. Lucas CE and Ledgerwood AM. "Prospective evaluation of hemostatic techniques for liver injuries". Journal of Trauma 16.6 (1976): 442-451. 
9. Walt AJ. "Founder's lecture: the mythology of hepatic trauma: or Babel revisited". The American Journal of Surgery 135.1 (1978): 12-18.

10. Elerding SC., et al. "Fatal hepatic hemorrhage after trauma". The American Journal of Surgery 138.6 (1979): 883-888.

11. Feliciano DV., et al. "Intra-abdominal packing for control of hepatic hemorrhage: a reappraisal”. Journal Trauma 21.4 (1981): 285-290.

12. Svoboda JA., et al. "Severe liver trauma in the face of coagulopathy. A case for temporary packing and early reexploration". The American Journal of Surgery 144.6 (1982): 717-721.

13. Burch JM., et al. "Abbreviated laparotomy and planned reoperation for critically injured patients". Annals of Surgery 215.5 (1992): 476-483.

14. Cue JI., et al. "Packing and planned reexploration for hepatic and retroperitoneal hemorrhage: critical refinements of a useful technique". Journal Trauma 30.8 (1990): 1007-1011.

15. Morris JA Jr., et al. "The staged celiotomy for trauma. Issues in unpacking and reconstruction". Annals of Surgery 217.5 (1993): 576-584.

16. Balogh ZJ., et al. "Postinjury abdominal compartment syndrome: from recognition to prevention". Lancet 384.9952 (2014): 1466-1475.

17. Watson JJ., et al. "Damage control laparotomy utilization rates are highly variable among level I trauma centers: pragmatic, randomized optimal platelet and plasma ratios findings". Journal of Trauma and Acute Care Surgery 82.3 (2017): 481-488.

18. Roberts DJ., et al. "Indications for use of damagecontrol surgery and damage control interventions in civilian trauma patients: ascoping review". Journal of Trauma and Acute Care Surgery 78.6 (2015): 1187-1196.

19. Kaafarani HM and Velmahos GC. "Damage control resuscitation in trauma". Scandinavian Journal of Surgery 103.2 (2014): 81-88.

20. Rajasekhar A., et al. "Survival of trauma patients after massive red blood cell transfusion using a high or low red blood cell to plasma transfusion ratio". Critical Care Medicine 39.6 (2011): 1507-1513.
21. Mizobata Y. "Damage control resuscitation: a practical approach for severely hemorrhagic patients and its effects on trauma surgery". Journal of Intensive Care 5 (2017): 4.

22. Duchesne JC., et al. "Damage control resuscitation in combination with damage control laparotomy: a survival advantage". Journal of Trauma 69.1 (2010): 46-52.

23. Ball CG., et al. "The impact of a massive transfusion protocol on major hepatic injuries: does it increase abdominal wall closure rates?". Canadian Journal of Surgery 56.5 (2013): E128-E134.

24. Bradley M., et al. "Damage control resuscitation protocol and the management of open abdomens in trauma patients". American Surgeon 80.8 (2014): 768-775.

25. Shrestha B., et al. "Damage-control resuscitation increases successful non-operative management rates and survival after severe blunt liver injury". Journal of Trauma and Acute Care Surgery 78.2 (2015): 336-341.

26. Duchesne JC., et al. "Damage control resuscitation: the new face of damage control”. Journal of Trauma 69.4 (2010): 976-990.

27. Jansen JO., et al. "Damage control resuscitation for patients with major trauma”. BMJ 338 (2009): b1778.

28. Bickell WH., et al. "Immediate versus delayed fluidresuscitation for hypotensive patients with penetrating torso injuries". The New England Journal of Medicine 331 (1994): 1105-1109.

29. Morrison CA., et al. "Hypotensive resuscitation strategy reduces transfusion requirements and severe postoperative coagulopathy in trauma patients with hemorrhagic shock: preliminary results of a randomized controlled trial". Journal of Trauma 70.3 (2011): 652-663.

30. Chatrath V., et al. "Fluid management in patients with trauma: restrictive versus liberal approach". Journal of Anaesthesiology Clinical Pharmacology 31.3 (2015): 308-316.

31. Carrick MM., et al. "Intraoperative hypotensive resuscitation for patients undergoinglaparotomy or thoracotomy for trauma: early termination of a randomizedprospective clinical trial". Journal of Trauma and Acute Care Surgery 80.6 (2016): 886-896.

32. Morrison CA., et al. "Hypotensive resuscitation strategy reduces transfusion requirements and severepostoperative coagulopathy in trauma patients with hemorrhagic shock:preliminary results of a randomized controlled trial". Journal of Trauma 70.3 (2011): 652-663. 
33. Schreiber MA., et al. "A controlled resuscitation strategy is feasible and safe in hypotensivetrauma patients: results of a prospective randomized pilot trial". The Journal of Trauma and Acute Care Surgery 78.4 (2015): 687-695.

34. Natthida Owattanapanich et al. "Risks and benefits of hypotensive resuscitation in patients with traumatic hemorrhagic shock: a meta-analysis". Scandinavian Journal of Trauma, Resuscitation and Emergency Medicine 26.1 (2018): 107.

35. Kaussen T., et al. "Recognition and management of abdominal compartment syndrome among German pediatric intensivists: results of a national survey". Annals of Intensive Care 2.1 (2012): S8.

36. Z hou JC., et al. "Current recognition and management of intra-abdominal hypertension and abdominal compartment syndrome among tertiary Chinese intensive care physicians". Journal of Zhejiang University Science B 12.2 (2011):156-162.

37. Wise R., et al. "Awareness and knowledge of intra-abdominal hypertension and abdominal compartment syndrome: results of an international survey". Anaesthesiology Intensive Therapy 47.1 (2015): 14-29.

38. Kirkpatrick AW., et al. "Spill your guts! Perceptions of Trauma Association of Canada member surgeons regarding the open abdomen and the abdominal compartment syndrome". Journal of Trauma 60.2 (2006): 279-286.

39. Kirkpatrick AW., et al. "Intra-abdominal hypertension and the abdominal compartment syndrome: updated consensus definitions and clinical practice guidelines from the World Society of the Abdominal Compartment Syndrome". Intensive Care Medicine 39.7 (2013): 1190-1206.

40. Balogh ZJ., et al. "Postinjury abdominalcompartment syndrome: from recognition to prevention". Lancet 384.9952 (2014): 1466-1475.

41. Morris JA Jr., et al. "The staged celiotomy for trauma. Issues in unpacking and reconstruction". Annals of Surgery 217.5 (1993): 576-584.

42. Regner JL., et al. "Surgical strategies for management of the open abdomen". World Journal of Surgery 36.3 (2012): 497510.
43. De Waele JJ and Leppäniemi AK. "Temporary abdominal closure techniques". The American surgeon 77.1 (2011): S46-S50.

44. Stone HH., et al. "Management of acute full thickness losses of the abdominal wall". Annals of Surgery 193.5 (1981): 612-618.

45. Acosta S., et al. "Multicentre prospective study of fascial closure rate after open abdomen with vacuum and mesh-mediated fascial traction". British Journal of Surgery 98.5 (2011):735743.

46. Cothren CC., et al. "One hundred percent fascial approximation with sequential abdominal closure of the open abdomen". The American Journal of Surgery 192.2 (2006): 238-242.

47. Aydin C., et al. "The effect of different temporary abdominal closure techniques on fascial wound healing and postoperative adhesions in experimental secondary peritonitis". Langenbeck's Archives of Surgery 393.1 (2008): 67-73.

48. Fernandez L., et al. "Temporary intravenous bag silo closure in severe abdominal trauma”. Journal of Trauma 40.2 (1996): 258-260.

49. Cheatham ML., et al. "Long-term physical, mental, and functional consequences of abdominal decompression". Journal of Trauma 56.2 (2004): 237-241.

50. Cheatham ML and Safcsak K. "Long-term impact of abdominal decompression: a prospective comparative analysis". Journal of the American College of Surgeons 207.4 (2008):573-579.

\section{Volume 2 Issue 6 August 2019 (C) All rights are reserved by Abdelkader Boukerrouche.}

